This item was submitted to Loughborough's Research Repository by the author.

Items in Figshare are protected by copyright, with all rights reserved, unless otherwise indicated.

\title{
Inherent asymmetry of the current output in a triboelectric nanogenerator
}

PLEASE CITE THE PUBLISHED VERSION

https://doi.org/10.1016/j.nanoen.2020.105045

PUBLISHER

Elsevier

VERSION

AM (Accepted Manuscript)

PUBLISHER STATEMENT

This paper was accepted for publication in the journal Nano Energy and the definitive published version is available at https://doi.org/10.1016/j.nanoen.2020.105045

\section{LICENCE}

CC BY-NC-ND 4.0

\section{REPOSITORY RECORD}

Dharmasena, Ishara. 2020. "Inherent Asymmetry of the Current Output in a Triboelectric Nanogenerator". Loughborough University. https://hdl.handle.net/2134/12585200.v1. 


\section{Inherent Asymmetry of the Current Output in a Triboelectric Nanogenerator}

2

\section{R D I G Dharmasena*}

Wolfson School of Mechanical Electrical and Manufacturing Engineering, Loughborough University, Loughborough, Leicestershire, LE11 3TU, United Kingdom.

E-mail: r.i.dharmasena@1boro.ac.uk

Abstract: Triboelectric Nanogenerators (TENGs) are a leading candidate in powering the next generation of flexible and mobile electronics. However, the sporadic variations of output current and the asymmetry (variations in magnitude) between the current peaks are some of the key issues of this technology, impeding the reliable operation, consistency and the reproducibility of their outputs. Investigating the causes for this asymmetric and sporadic behaviour is therefore critical in developing optimised and TENG devices. This study, for the first time, comprehensively investigates the asymmetry between the positive and negative current peaks (corresponding to ascending and descending motion half cycles) and the underlying reasons for this phenomenon. The operation of the TENG is studied in contact and non-contact operating modes, revealing a number of new findings on the current output generation and its optimisation, in order to minimize their peak variations and to enhance the power output. Finally, the significance of this study on TENGs for self-powered sensing, energy harvesting, device characterisation and standardisation is discussed, leading towards sustainable future applications.

Keywords: Energy Harvesting, Triboelectric Nanogenerators, Current Peaks, Asymmetric Outputs, Adhesion induced impulsive separation.

\section{Introduction}

Triboelectric Nanogenerators (TENGs) are one of the most promising upcoming technologies to power the next generation of flexible and wearable low-power electronics.[1-6] TENGs offer a wide range of advantages such as light weight, low cost, flexibility, durability, high instantaneous power density and power conversion efficiency etc., over other mechanical energy harvesting techniques, especially at relatively low frequencies related to ambient 
mechanical movements including human motion.[1][7] These devices have been demonstrated as energy harvesting devices as well as self-powered sensors, in a wide variety of applications.[4,8-11]

Among many challenges of this technology, the inconsistency and variability of the electrical outputs is a key limitation that disrupts the development of sustainable TENG applications. Current outputs of a TENG are typically inconsistent, producing sporadic output peaks.[1][4] More specifically, there exists a significant variation between the peak current outputs of a TENG during ascending and descending motion cycles (hereafter referred to as asymmetry between peaks) (Fig. 1a-f), even under consistent motion inputs.[12-14] Such asymmetric current outputs are specifically observed in the TENG designs which belong to the vertical charge polarization TENG (VCPTENG) category, which depend on the vertical contact and separation movement of triboelectric layers like the vertical contact-separation mode TENG [12-18]. This asymmetry could adversely affect the performance and the reliability of a TENG, particularly in self-powered sensing applications which include the measurement of motion parameters such as velocity, acceleration, trajectory (Fig. 1d) [15,19,20], and, in applications such as sensing chemicals (Fig. 1e) [16][21] and ultraviolet (UV) radiation (Fig.

(a)

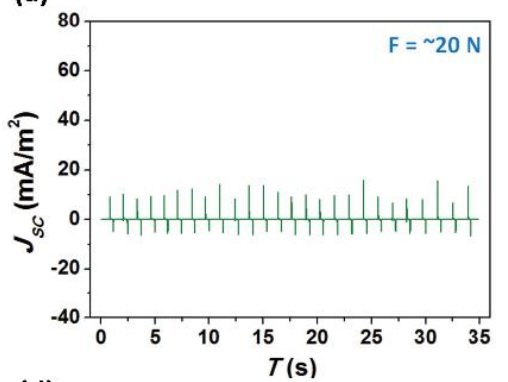

(d)

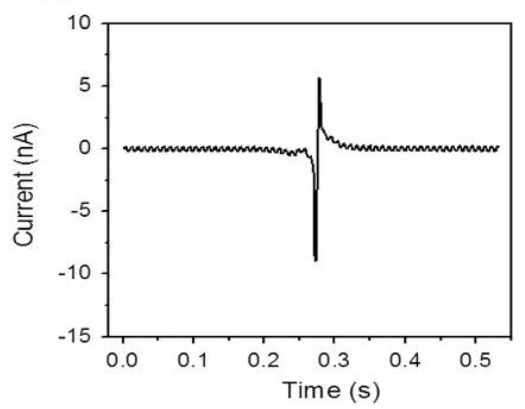

(b)

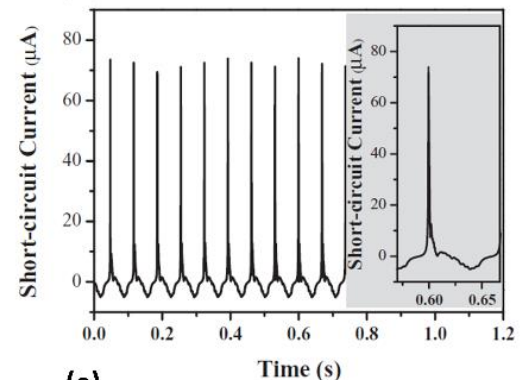

(e)

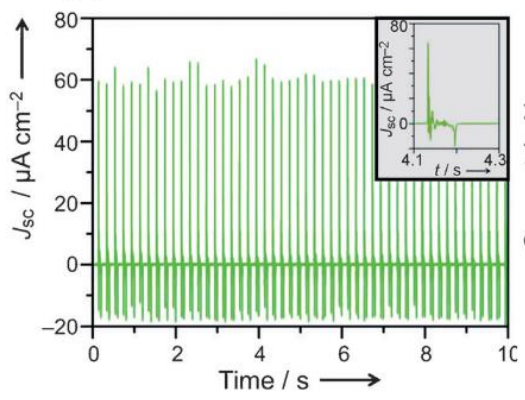

(c)

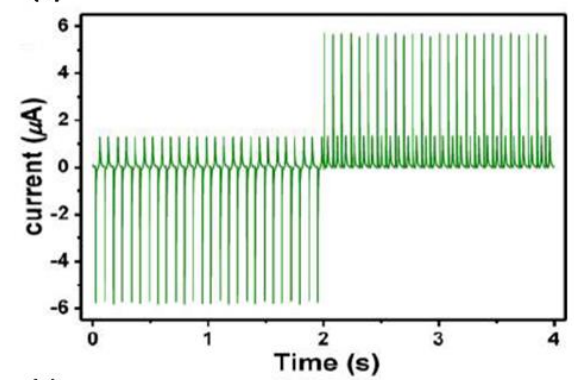

(f)

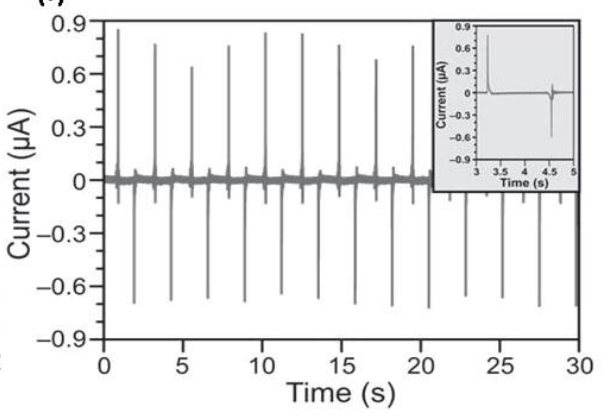

Fig. 1: Few examples for the asymmetric and irregular current outputs of TENGs presented in the literature. (a) Current density plotted against time for a vertical contact separation TENG (VCSTENG), as reported by Wang et al. [12]. Copyright 2014, John Wiley and Sons. (b) Short circuit current plotted against time for a spring assisted VCSTENG, reported by Chen et al. [22]. Copyright 2013, John Wiley and Sons (c) Outputs of a VCSTENG, demonstrating the current before (left) and after (right) rectification, as reported by Zhu et al. [14]. Copyright 2012, American Chemical Society. Asymmetric current outputs of several sensor units reported in the literature demonstrating; (d) Current output of a velocity sensor, reported by Han et al. [15]. Copyright 2014, Elsevier. Current output of (e) self-powered mercury ion sensor in response to the ion concentration, reported by Lin et al. [16] (Copyright 2013 John Wiley and Sons), and, (f) self-powered UV sensor reported by Lin et al. [17] (Copyright 2014, John Wiley and Sons). 
1f) [17]. Moreover, the asymmetry between positive and negative current peaks cause varying power output pulses, disturbing the consistency of the TENGs when used for energy harvesting. While the reason for the asymmetry between positive and negative current peaks can potentially be attributed to the velocity differences during ascending and descending motion cycles[22], there is a lack of in-depth experimental and theoretical studies on this phenomenon.

This paper presents a study on the asymmetric nature of the current outputs of a TENG, observed during ascending (contact) and descending (separation) motion cycles. A theoretical analysis based on the distance dependent electric field (DDEF) model is presented, evaluating the critical parameters affecting the outputs. This is followed by an experimental analysis, where a uniform motion input (defined as a symmetric motion profile during ascending and descending movements, for example sinusoidal motion) is utilised to observe the practical behaviour of the current generation. The evaluation of the current outputs is done in two different stages; the short circuit stage, and, under increasing load conditions, revealing a number of novel insights to the behaviour of a TENG. Furthermore, criteria to manage variation of the current and power outputs is presented in the process.

\section{Analysis of the Short Circuit Outputs}

Short circuit current $\left(I_{S C}\right)$ of a TENG is an important parameter specially in sensing applications, and, to evaluate and compare the electrical performance of a TENG. In this section, the intrinsic asymmetry of the $I_{S C}$ outputs are analysed in different TENG operating regimes.

\subsection{TENG Current Generation: Fundamentals}

A typical TENG would consist of two dielectric contact surfaces, and, two electrodes on the opposite sides of the contacting surfaces (Fig. 2a) [23][24]. Static charging occurs on the TENG contact surfaces due to the triboelectric effect, and these charges would remain for relatively long durations of time [25]. The relative movement of such charged surfaces induces a potential difference between the electrodes, causing an output current between them. In 2017, we presented the distance dependent electric field (DDEF) model, successfully explaining the working principles and output trends of the TENGs.[23,26-28] Herein, the DDEF model is used to further understand this current generation behaviour. 
The DDEF model utilises the distance-dependent variation of the electric fields of the

triboelectric layers and the electrodes, to define their electrical output behaviour. Considering

a rectangular charged surface with dimensions $L$ and $W$, and a uniform surface charge density $\sigma$, the DDEF model defines the overall electric field perpendicular to this charged surface $\left(E_{z}\right)$

as:

$$
E_{z}=\frac{\sigma}{\pi \varepsilon} \arctan \left(\frac{L / W}{2(z / W) \sqrt{4(z / W)^{2}+(L / W)^{2}+1}}\right)=\frac{\sigma}{\pi \varepsilon} f(z)
$$

where $z$ is the distance from the charged surface, and $\varepsilon$ is the permittivity of the medium[23].

The device structure used for the theoretical and experimental work in this study is a vertical

contact-separation mode TENG (VCSTENG) which is a subcategory of VCPTENG,

consisting of polydimethylsiloxane (PDMS) and polyethylene terephthalate (PET) contact

(a)

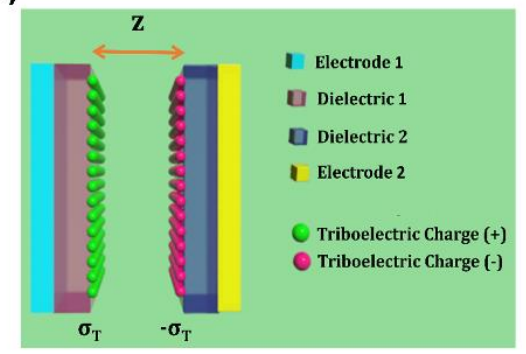

(d)

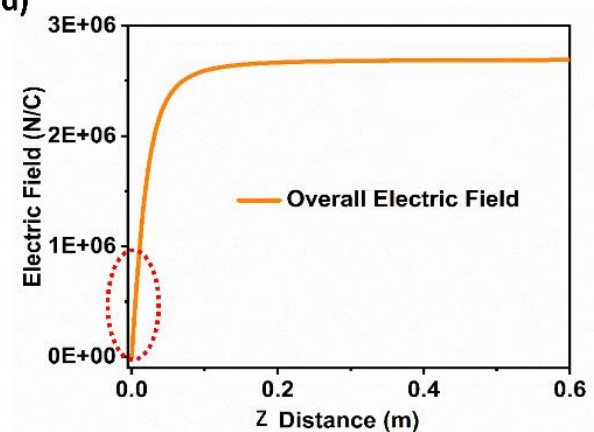

(b)

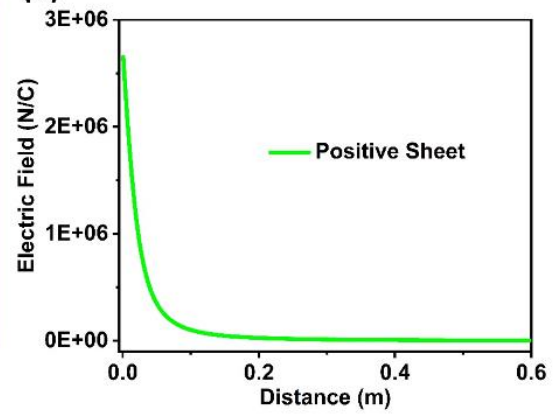

(c)

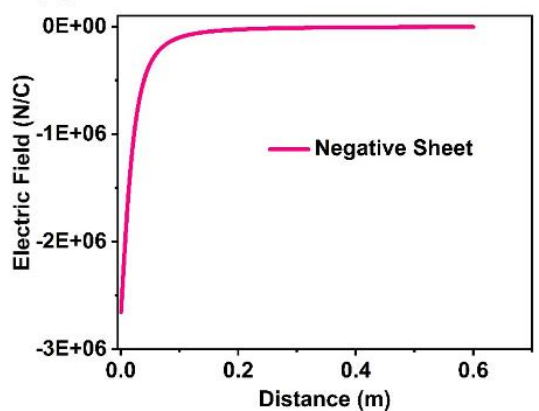

(e)

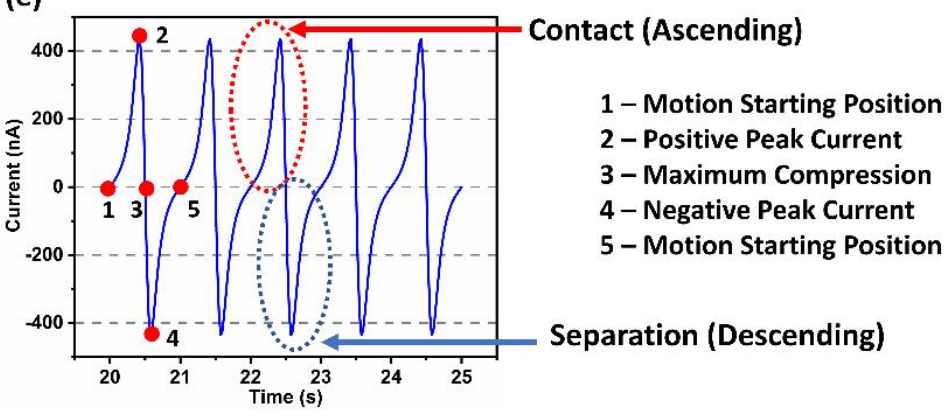

Fig. 2: Fig. 2: (a) Schematic of the structure of a TENG, indicating the dielectric layers, electrodes, triboelectric charge densities $\left(\sigma_{T^{+}}{ }^{+}, \sigma_{T}{ }^{-}\right)$, and TENG layer separation (z). DDEF model simulations for the electric field variation against the distance from the surface for a triboelectrically charged (b) positive, and, (c) negative TENG sheets (dimensions are parameters described in supplementary note 1). (d) DDEF model simulations for the electric field variation at the positive electrode (electrode 1) of the TENG, against the distance (z) between the charged TENG surfaces. Region with the high electric field gradient is shown highlighted. (e) DDEF model simulations for the short circuit current of the TENG, under $1 \mathrm{~mm}$ amplitude, $1 \mathrm{~Hz}$ frequency sinusoidal movement. A current half cycle corresponding to the ascending movement is marked with a red circle, and a current half cycle corresponding to descending movement marked with a blue circle. Some of the significant points of the current cycle corresponding to the first contact-separation movement cycle are denoted using markers 1 to 5 . 
surfaces (Supplementary Note 1) [23][26]. The selection of a VCSTENG device structure for this study is based on several factors. Firstly, VCSTENG is one of the most widely used structures for TENG prototypes and applications.[24][23] Secondly, the asymmetry of outputs is commonly observed related to VCSTENG devices, much more prominent compared to TENG categories such as sliding mode TENGs.[18] Finally, VCSTENG represents the simplest demonstration of VCPTENGs, therefore, by extension, the outcomes of this study are potentially be applicable to other VCPTENG device types.

The DDEF model was used to simulate the output trends of this device (Fig. 2), to identify the important regions of the output current cycle. Firstly, considering the PET (positively charged) sheet, the overall electric field against the distance from its surface can be demonstrated using eqn. (1) (Fig. 2b). Similarly, the corresponding electric field originating from the PDMS (negatively charged) sheet is depicted in Fig. 2c.[23][27][26] Now, considering the TENG arrangement shown in Fig. 2a, the overall electric field acting at the electrode interfaces is influenced by both positive and negative triboelectric surfaces, and, their respective distance to the electrodes. For instance, when the separation between the TENG surfaces (z) increases, the overall electric field at the positive electrode (dielectric 1 electrode 1 interface) is demonstrated in Fig. 2d. Herein, as z increases, the overall electric field at this interface increases positively, therefore, electrode 1 develops a positive potential (a similar negative potential is developed at the negative electrode). The potential difference between the electrodes creates a current flow between the two electrodes. On the other hand, when $\mathrm{z}$ is decreased, a current flow in the reverse direction can be observed. Therefore, an induced alternating current output corresponding to $\mathrm{z}$ can be observed from the TENG. This current output is proportional to the electric field gradient [29][23], therefore, Fig. 2d suggests that rapid variations in overall output current can be expected closer to the contact position of triboelectric surfaces [27]. 
The exact nature of this output current can be simulated using the DDEF model, in the contact-separation regime. Herein, the short circuit output charge density $\left(\sigma_{U}\right)$ for the TENG is given by:

$$
\sigma_{U}=\frac{\sigma_{T}\left[\frac{1}{\varepsilon_{1}} \int_{x_{1}}^{x_{1}+z} f(x) d x+\frac{1}{\varepsilon_{2}} \int_{x_{2}}^{x_{2}+z} f(x) d x\right]}{\left(\frac{1}{\varepsilon_{1}}+\frac{1}{\varepsilon_{2}}\right) \int_{0}^{x_{1}+x_{2}+z} f(x) d x}
$$

therefore, the short circuit current $\left(I_{S C}\right)$ is obtained using,

$$
I_{S C}=\mathrm{A} J_{S C}=\mathrm{A} \frac{d \sigma_{U}}{d t}
$$

where, $x_{1}$ and $x_{2}$ represent the thicknesses, $\varepsilon_{1}$ and $\varepsilon_{2}$ are permittivities, $\mathrm{A}$ is the surface area of tribelectric layers, $t$ is time, and, $J_{S C}$ is the output current density.

Using eqn. (2) and (3), the simulated $I_{S C}$ for the TENG (subjected to $1 \mathrm{~mm}$ amplitude, $1 \mathrm{~Hz}$ frequency sinusoidal motion) is depicted in Fig. 2e. Considering this simulation, in the given arrangement, the movement of the TENG layers begin with their maximum separation (equation S1 of supplementary note 1) which is marked as the motion starting position, and the corresponding current output is zero (indicated as marker 1). The maximum positive peak current is observed at marker 2 . As the TENG layer ascends to the contact position ( $\mathrm{z}=0$, which is equivalent to the maximum compression of TENG layers in the experimental scenario), the current output drops to zero (marker 3) since the TENG layer movement halts momentarily to reverse its direction following the sinusoidal motion cycle. A negative peak current (marker 4) is observed during the descending motion cycle of the TENG, and the current output returns to zero again as the direction of movement is about to change (marker 5). Therefore, as per the simulation results, the ascending movement of the TENG layers correspond to the markers $1-3$, whereas the descending movement of the TENG layers correspond to markers $3-5$. A similar pattern is repeated over rest of the current cycles.

\subsection{TENG Current Generation: Contact Mode}

Fig. 3a depicts the DDEF model simulations for the $I_{S C}$ of the TENG, under identical conditions to that of Fig. 2e, where the TENG is subjected to a sinusoidal contact-separation 
(a)
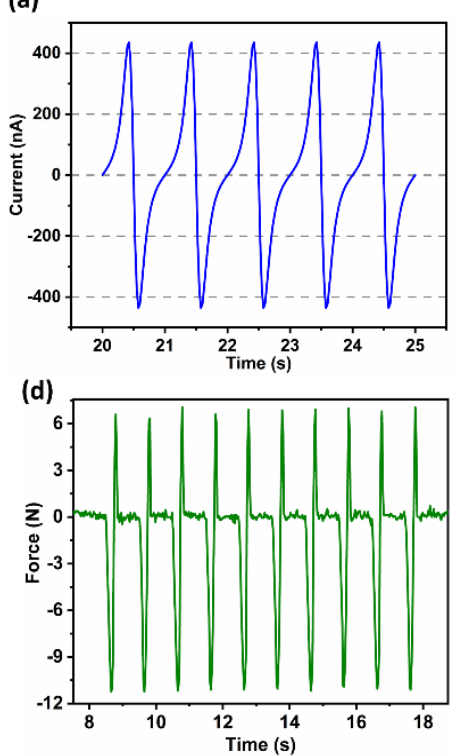

(b)

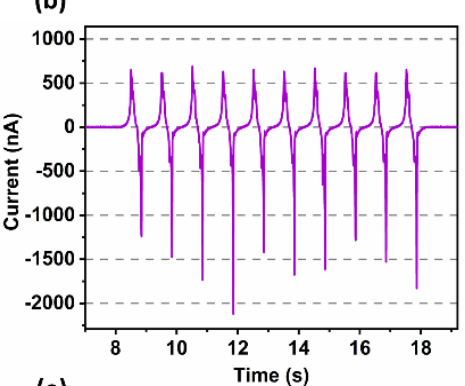

(e)

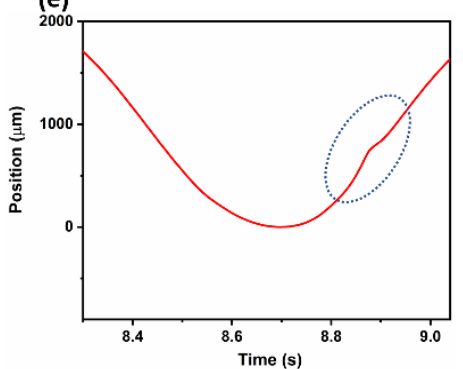

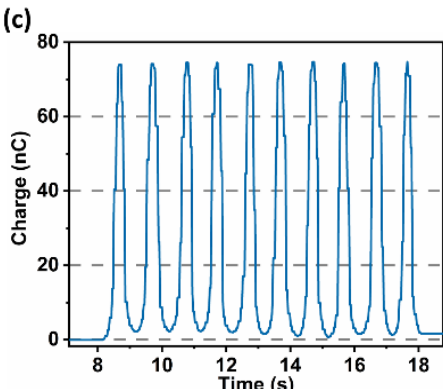

(f)

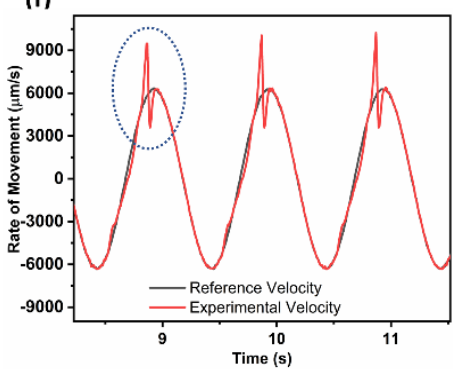

(g)

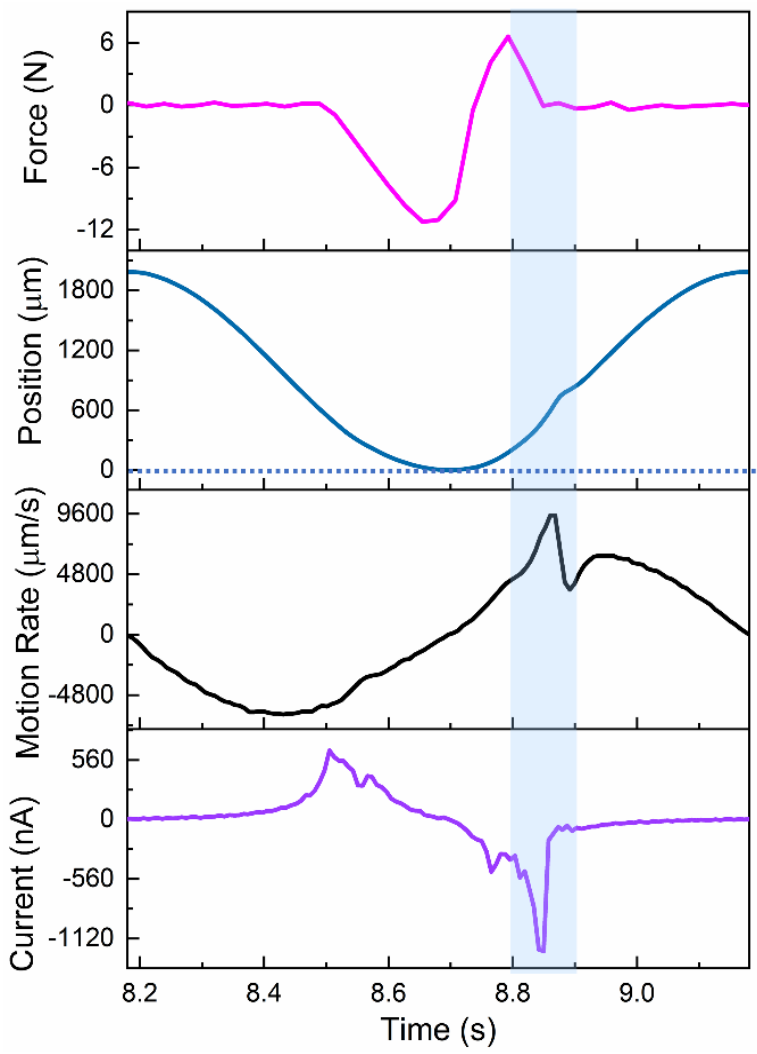

Fig. 3: (a) DDEF model simulations for the Isc vs time for the TENG $(1 \mathrm{~mm}$ amplitude and $1 \mathrm{~Hz}$ frequency sinusoidal movement), and, Corresponding experimental outputs for (b) Isc (c) Qsc, and, (d) contact force. The parameters of the stage connected to the moving TENG layer, depicting (e) position, and, $(f)$ velocity, plotted against time. (g) Experimental outputs for the contact force, position, motion rate and the corresponding current peak of the TENG, demonstrated with respect to a common time scale. 
descending/separation movement) are predicted to be similar in nature with equal peak values, relatively similar time durations, and, equivalent shapes.

An experimental analysis of this device was carried out under similar conditions, using the motion control system depicted in Supplementary Note 2.[23][26] The motion of the TENG layer was controlled using a moving stage connected to a high precision voice coil actuator, while the position of the stage, contact force between TENG layers, and the electrical outputs of the TENG including current and charge were recorded. The experimental $I_{S C}$ of the TENG is demonstrated in Fig. 3b, which deviates from the simulated outputs in terms of the asymmetry between positive and negative current half cycles. As depicted in Fig. 3b, the positive current half cycles are consistent with relatively similar peak values. However, the negative current half cycles record significantly higher peak values, with notable variations even among the negative peaks. This behaviour is consistent with the previous experimental observations for TENGs as depicted in Fig. 1, where a significant variation between positive and negative current half cycles is observed. Comparing with the simulation results, there appears to be an interaction between the TENG layers during the contact stage, which potentially alters the motion profile of the TENG layers.

To further analyse this scenario, the experimental short circuit charge output $\left(Q_{S C}\right)$ of the TENG was recorded (measured relative to the motion starting condition), as depicted in Fig. 3c. When the TENG layers are ascending, Qsc increases, and maximizes at the reference contact position (maximum compression, as described in supplementary note 2) of TENG layers, resulting in an overall maximum charge of $\sim 75 \mathrm{nC}$. During the descending movement, $Q_{S C}$ decreases and returns towards zero. Considering the individual charge peaks, $Q_{S C}$ increases and decreases by similar amounts, indicating that roughly the same amount of output charge is being transferred back and forth between the electrodes during ascending and descending movements of TENG surfaces. Owing to the relatively fixed amount of charge transfer, factors such as significant charge leakages can be neglected, therefore, the 
asymmetry between positive and negative current outputs lead to the possibility of an interaction taking place between the TENG surfaces during their contact phase which alters the motion rate (velocity of movement) of the TENG layers.[27] As described in the Supplementary Note $2[23,26,27]$, in the experimental setup, one of the TENG layers is stationary with a load cell on the back side, whereas the second TENG layer is connected to the moving stage operated by the voice coil actuator. When the moving TENG layer comes into contact with the stationary TENG layer, a compressive force will be recorded by the load cell. As described in Supplementary Note 2 and our previous work $[23,26,27]$, the position of the moving stage which records $\sim 10-11 \mathrm{~N}$ of force on the load cell was considered as the reference contact position (zero separation position) during these experiments. Analysing the force recordings (Fig. 3d), each compressive force peak (denoted with a negative force value) corresponds to a contact between the TENG layers. During each of these compressions, a maximum compressive force of $\sim 11 \mathrm{~N}$ was applied. It is important to notice that, following each compression peak, and, prior to consequent relaxation of load cell, a positive force peak of around $6 \mathrm{~N}$ is detected (Fig. 3d). This indicates a considerable tensile force applied on the load cell during the separation half-cycle, just before the two TENG layers are separated.

From this information, it can be hypothesized that, during the initial phase (corresponding to markers 1 to 3 in Fig. 2e), TENG layers are subjected to ascending movement half-cycle, and these layers are contacted under a compressive force. While the triboelectric surfaces are in contact, they develop an adhesion or a "sticking effect", potentially due to electrostatic forces caused by opposite triboelectric charges on the surfaces, Van der Waals forces, or other related surface interactions.[30-32] Consequently, during the onset of the separation phase (descending movement half-cycle), the compression force is removed via the motion control system, as indicated through a reduction in the negative force in Fig. 3d. However, the adhesion between the triboelectric surfaces is still prevalent, therefore, the moving TENG 
surface drags the stationary TENG surface away from the load cell, resulting in a tensile force, as indicated through the positive force peak. As the moving stage continues the separation movement exerting a force larger than the adhesive forces, TENG layers are subjected to a sudden impulsive separation, as indicated by the rapid drop in the positive force peak of the load cell (Fig. 3d). This characteristic phenomenon alters the original (input) motion profile of the system.

Fig. 3e indicates the position data of the stage which carries the moving TENG layer, as recorded by the motion control system. In the given example, $0 \mu \mathrm{m}$ position acts as the contact position of the two TENG layers, and the motion controller moves between $2000 \mu \mathrm{m}$ (largest separation) position and $0 \mu \mathrm{m}$ position (reference contact position), producing a $1 \mathrm{~mm}$ amplitude, $1 \mathrm{~Hz}$ motion profile. Analysing this scenario closer to the contact position, there is a sudden deviation in the motion profile of the stage compared to a typical sinusoidal movement, caused by the impulsive separation of the TENG layers as highlighted in Fig. 3e. The time derivative of this motion profile representing the rate of the movement of TENG system (for 3 consecutive contact separation cycles) is depicted in Fig. 3f. A spike in the rate of movement of the TENG layer is visible for each motion cycle, corresponding to a similar time scale as the impulsive separation of the TENG layers. Comparing with the reference motion rate (velocity) profile (indicated in black), these deviations are clearly notable, as shown in Fig. 3f. Such increments in the rate of movement have been shown in previous theoretical and experimental studies to result in increased peak current outputs. $[1][23][27][26]$

In order to further analyse these outputs with respect to their time scale, $I_{S C}$, contact force, position and motion rate information corresponding to a single contact-separation cycle of the TENG were compared (considering the maximum compression of the TENG layers as the reference point) (Fig. 3g). As highlighted in Fig. 3g, the tensile force caused by the adhesion of the TENG layers is maximised at $\sim 8.8 \mathrm{~s}$, and a sudden separation of TENG layers is 
observed directly afterwards, causing the force value to rapidly decline and return to zero. The effect of this impulsive separation is recorded as a variation of movement of the stage and the consequent instantaneous peak in its motion rate, which results in the exaggerated negative $I_{S C}$ of the TENG. Fig. 3g provides strong evidence to the co-dependence of these phenomenon, which takes place at a corresponding time scale. Extending this analysis further, the motion profile recorded by the experimental scenario was used as the motion input for a corresponding DDEF model simulation, as demonstrated in Supplementary Note 3. This analysis also confirms the effect of adhesion induced impulsive separation on the asymmetry of TENG current output.

Therefore, it is clear that the inherent adhesion between the triboelectric surfaces cause the TENG layers to separate sporadically under an applied motion, causing an impulsive peak in its separation velocity. This phenomenon, herein referred to as the "adhesion induced impulsive separation", causes the current output corresponding to the separation (descending) movement to increase significantly compared to the current output corresponding to the contact (ascending) movement.

\subsection{TENG Current Generation: Non-Contact Mode}

The analysis presented in section 2.2 provides comprehensive evidence to the adhesion induced impulsive separation and its effect on the current output of a TENG. However, further evidence for this phenomenon can be obtained by operating the TENG in the noncontact motion regime. Herein, a similar motion profile $(1 \mathrm{~mm}$ amplitude $1 \mathrm{~Hz}$ frequency sinusoidal) is utilised, however, the initial distance between the TENG layers is increased by an additional $100 \mu \mathrm{m}$ (eqn. S2 of supplementary note 1). Hence, the motion controller moves the TENG layer between $100 \mu \mathrm{m}$ position and $2100 \mu \mathrm{m}$ position, therefore, leaving a $100 \mu \mathrm{m}$ minimum gap compared to the reference contact position $(0 \mu \mathrm{m}$ position $)$, which ensures that the TENG layers do not contact each other throughout their whole motion cycle. This would eliminate the adhesion induced impulsive separation by preventing the contact between the 

of $I_{S C}$ peaks observed in section 2.2 was purely due to the adhesion between the triboelectric
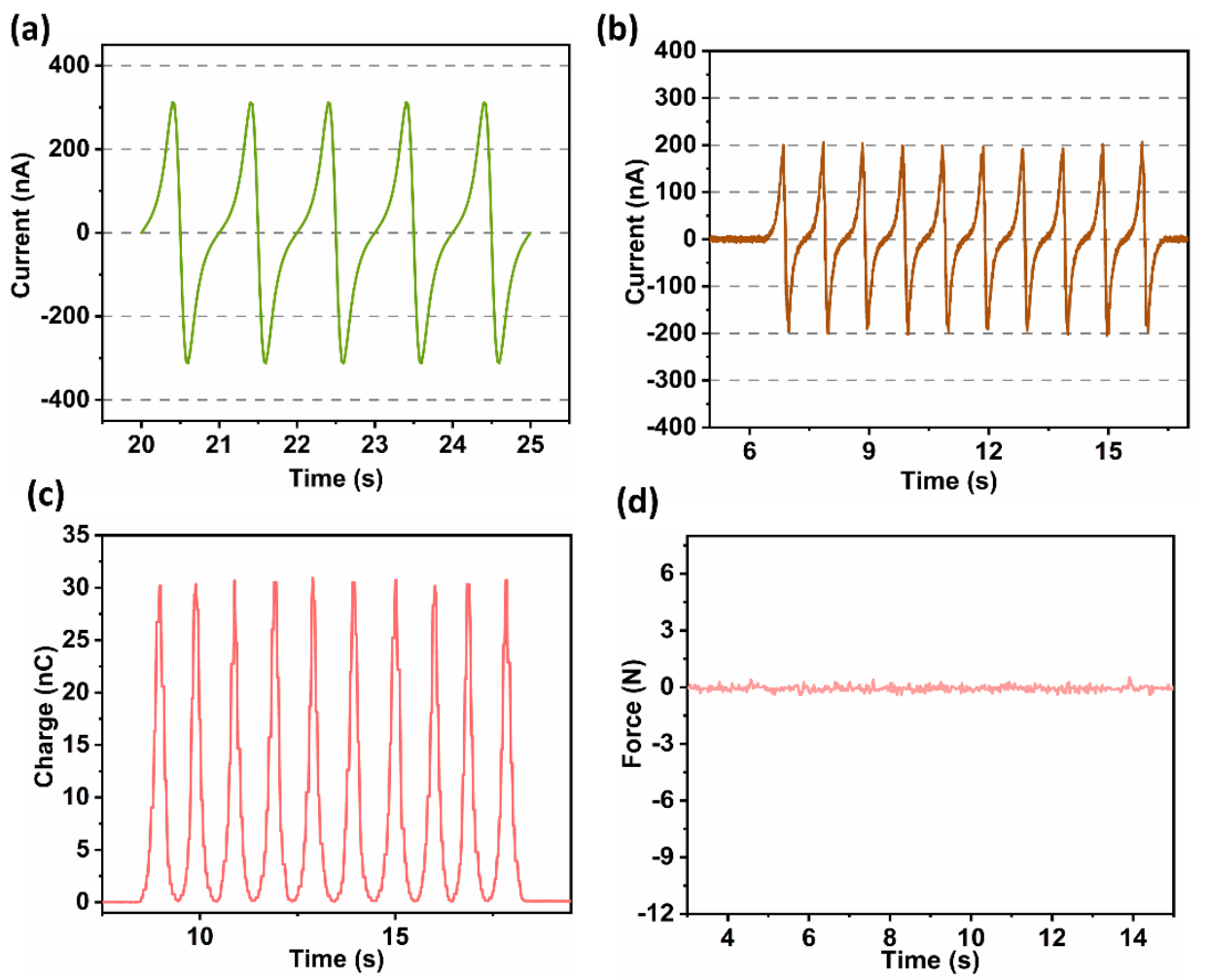

(d)

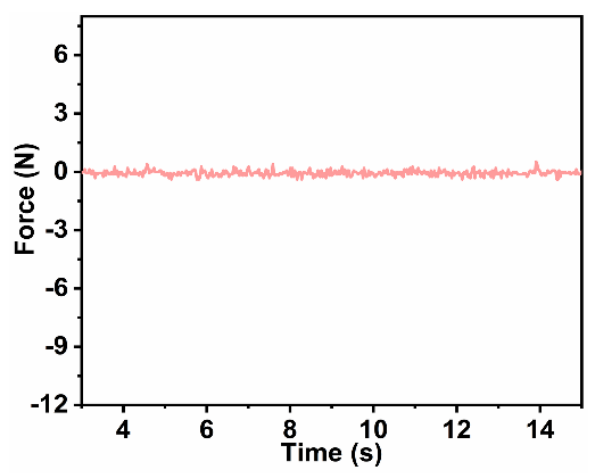

(e)

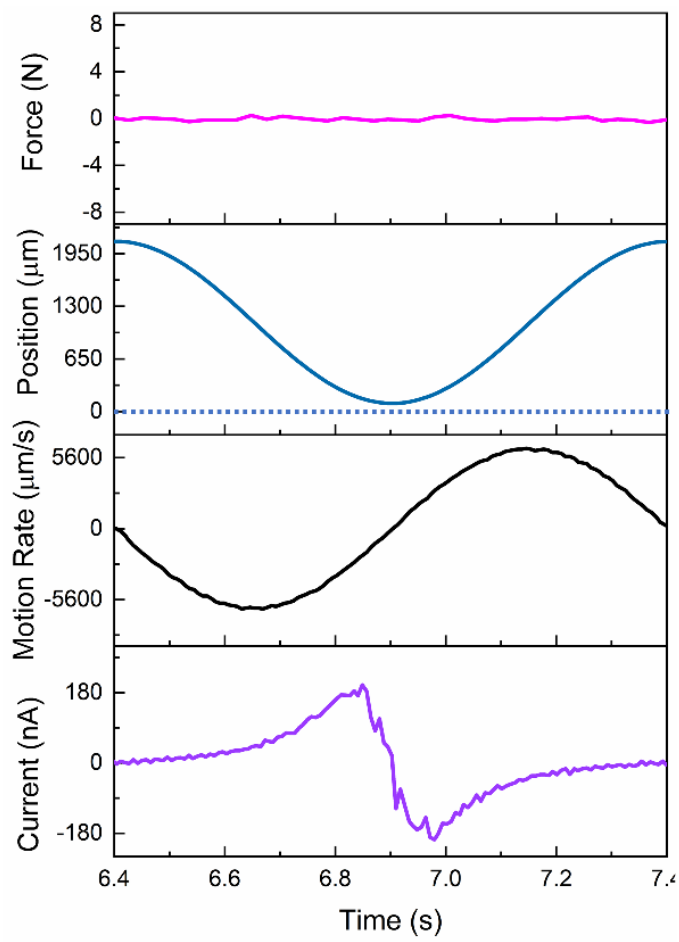

Fig. 4: The outputs of the TENG considering a non-contact motion regime (minimum separation of $100 \mu \mathrm{m}$ of the TENG layers with respect to the contact state), demonstrating (a) DDEF model simulations for the Isc, and, the corresponding experimental (b) Isc, (c) Qsc, and (d) contact force. (e) Experimental outputs related to the non-contact mode, including contact force, position, motion rate and the corresponding current peak of the TENG, demonstrated with respect to a common time scale. 
surfaces.

Fig. 4a depicts the DDEF model simulations for the $I_{S C}$ of the TENG under non-contact motion regime, predicting a symmetric current output comparable to the contact regime simulations. It is important to notice that the peak current values observed in the non-contact regime are relatively lower than the corresponding contact mode scenario. This is due to the lower output induction caused by the increased minimum separation (h) of TENG surfaces, as explained in our previous theoretical and experimental work.[27] The corresponding experimental $I_{S C}$ is indicated in Fig. $4 \mathrm{~b}$, and the positive and negative peaks are symmetric with equal amplitudes (peak values) observed for both current half cycles, contradicting with the experimental $I_{S C}$ behaviour of the contact mode (Fig. 3b). The $Q_{S C}$ of the TENG is demonstrated in Fig. 4c, indicating that similar amount of charge (overall maximum charge amount of $30 \mathrm{nC}$ ) is transferred between the electrodes during ascending and descending movement cycles. Furthermore, the load cell does not show any contact force during the ascending and descending movements, confirming that that the TENG layers are operating in the non-contact motion regime (Fig. 4d). This suggests that, when the TENG is operating in the non-contact regime, the adhesion between the triboelectric surfaces is avoided, preventing the adhesion induced impulsive separation. Fig. 4e plots the force, position, motion rate and current output corresponding to an ascending and descending motion cycle. Unlike the scenario in Fig. 3g, the TENG layers do not contact each other, therefore, the motion profile and the motion rate of the TENG layers are not altered significantly. Consequently, the current output corresponds to the simulated output, providing symmetric positive and negative peak values.

Considering the overall $I_{S C}$ behaviour, adhesion induced impulsive separation between the triboelectric surfaces becomes the key phenomenon causing the asymmetric output behaviour of the TENG current, when the motion input is symmetric and uniform.

\section{Analysis of the Current through Load Resistors}


(a)

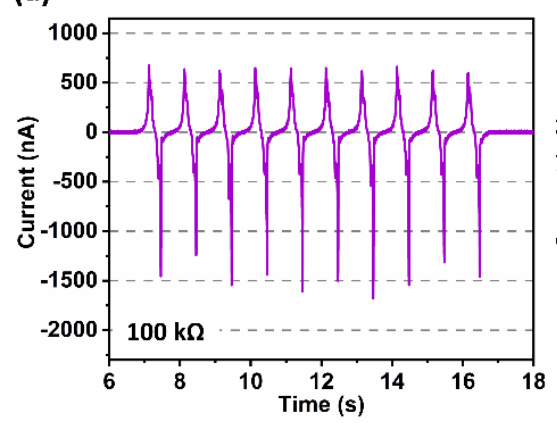

(d)

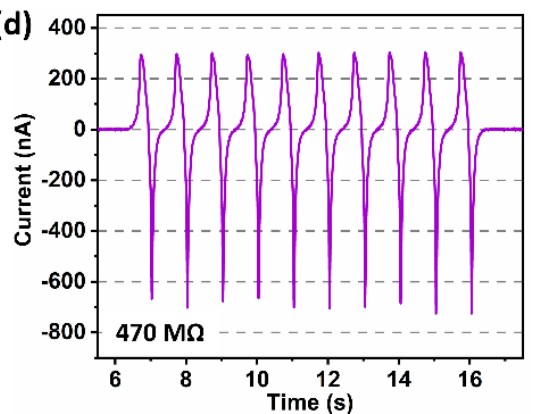

(b)

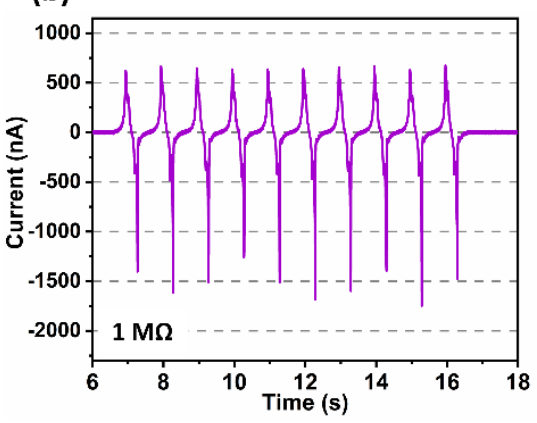

(c)

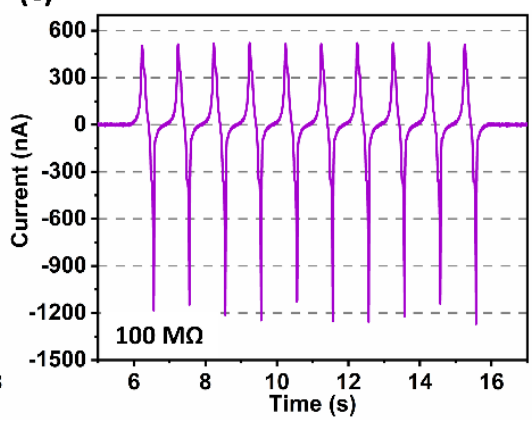

(e)

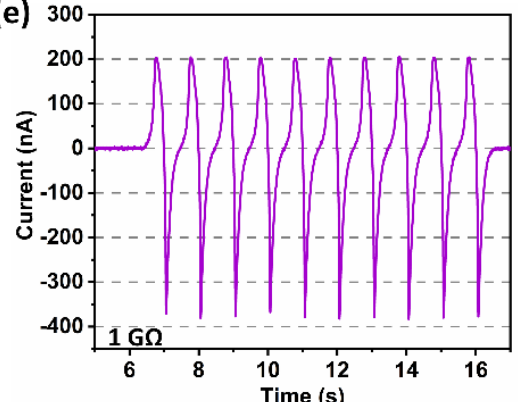

Fig. 5: The experimental TENG current outputs operating in the contact regime under a $1 \mathrm{~mm}$ amplitude, $1 \mathrm{~Hz}$ frequency sinusoidal motion profile, through loads of (a) $100 \mathrm{k} \Omega$, (b) $1 \mathrm{M} \Omega$, (c) $100 \mathrm{M} \Omega$, (d) $470 \mathrm{M} \Omega$, and, (e) $1 \mathrm{G} \Omega$

Considering the experimental output current of the TENG through different loads in noncontact regime, the current half-cycles demonstrate symmetric characteristics in comparison to the operation in the contact regime. This relatively symmetric behaviour is consistent 

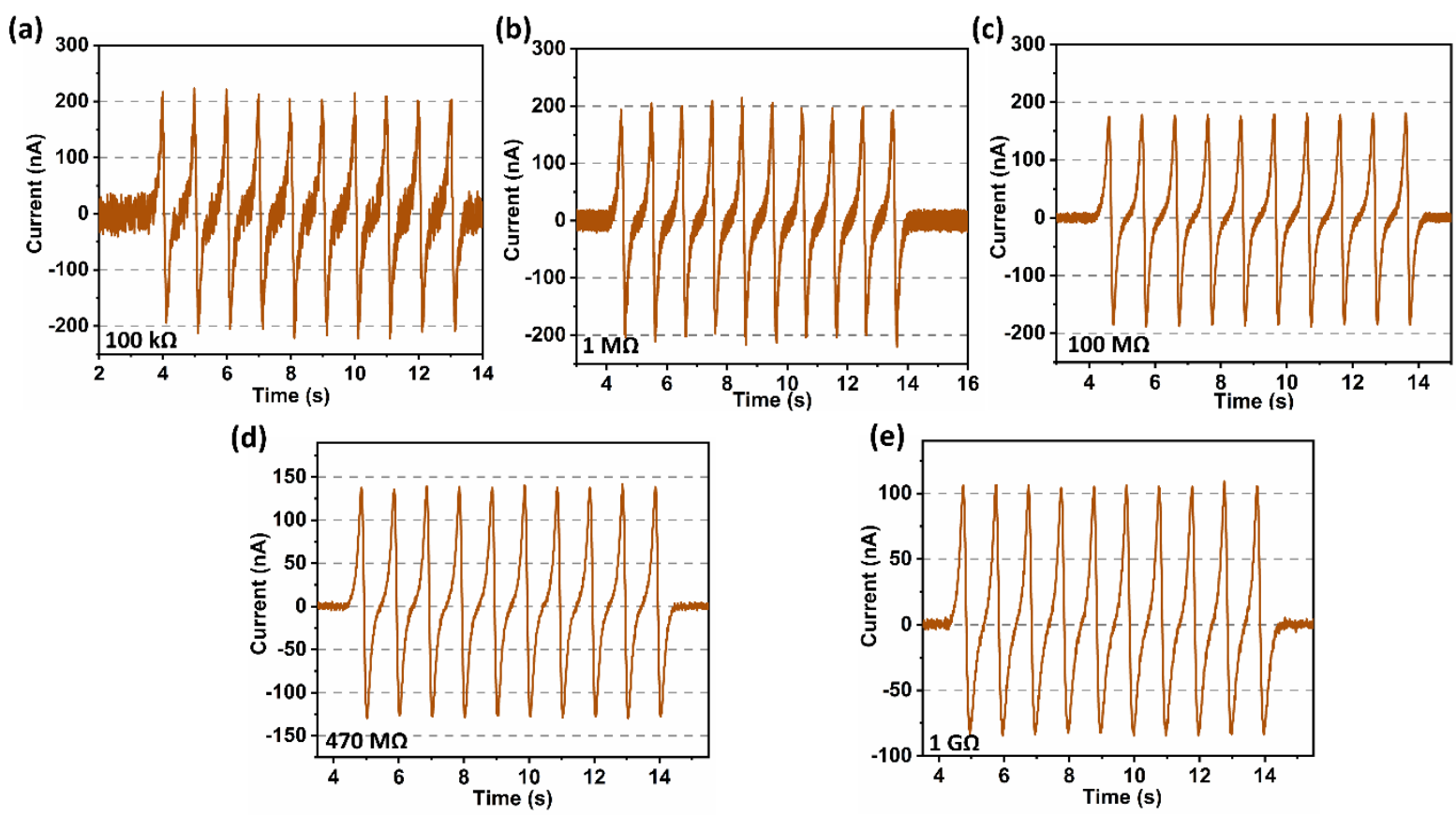

Fig. 6: The experimental TENG current outputs, under a $1 \mathrm{~mm}$ amplitude, $1 \mathrm{~Hz}$ frequency sinusoidal motion profile, subjected to a non-contact regime (minimum separation between TENG layers $=100 \mu \mathrm{m}$ ), through loads of (a) $100 \mathrm{k} \Omega$, (b) $1 \mathrm{M} \Omega$, (c) $100 \mathrm{M} \Omega$, (d) $470 \mathrm{M} \Omega$, and, (e) $1 \mathrm{G} \Omega$.

throughout most of loads tested in the experiment (Fig. 6a-e). As shown in section 2.3, the

triboelectric layers are not contacting each other during the non-contact mode operation,

therefore, the adhesion induced impulsive separation, which created in the asymmetry

between current peaks in the previous scenario, does not prevail in this situation. Despite the

symmetric nature of current peaks, the magnitude of the current outputs is relatively lower in the non-contact mode in comparison to the outputs of the TENG in contact mode, signalling a lower power generation.

The percentage variation of peak output power between positive and negative motion half cycles is depicted in Fig. S4 (Supplementary Note 4), plotted against corresponding load values. Herein, the difference between the average positive and negative current peaks is recorded, and, presented as a percentage value of the larger peak, to obtain an idea about the asymmetry of the output power. Fig. S4 indicates that the percentage variation of the power peaks corresponding to ascending and descending motion half cycles is almost $80 \%$ through most of the load values in the contact mode. However, in the non-contact mode operation, this variation is around $10 \%$ for most of the load values, confirming the higher consistency and 
symmetry of the power output in the non-contact mode operation, under a symmetric and uniform input motion profile.

\section{Discussion}

\subsection{Uniform Input Motion Profiles}

This study presented a fundamental analysis on the internal factors of a TENG which causes the asymmetry between the positive and negative current half cycles, considering a uniform input motion profile. Analysing the TENG output trends, it is evident that the adhesion induced impulsive separation is the inherent factor causing the asymmetry between the current peaks.

As evident from sections 2.2 and 2.3, The occurrence of this effect is strictly related to the contact between the triboelectric surfaces. Appreciating the fact that the motion input is a relatively uniform and symmetric motion profile (eg: sinusoidal motion), during the ascending (contact) phase, the triboelectric surfaces contact each other relatively smoothly, as depicted by the position (Fig. 3e) and velocity (Fig. 3f) profiles. This results in corresponding current peaks being uniform and predictable (Fig. 3b positive peaks). However, the intrinsic adhesion between the triboelectric surfaces following contact alters the input motion profile, and, results in an impulsive separation of the TENG layers (Fig. 3g), which increases the peak current of the corresponding current half cycle (in this case negative current). In addition to the asymmetry between positive and negative peaks, a variation within the negative peak values in different motion cycles is observed during this phase (Fig. 3b), which can be attributed to the abrupt nature of the TENG layer separation. Therefore, adhesion induced impulsive separation is the dominating internal phenomenon of a TENG, which affects the symmetry of the current output in the contact regime.

On the other hand, operating the TENG in non-contact regime eliminates the asymmetry between the positive and negative current half cycles, especially in low load conditions. Noncontact mode prevents the contact between the triboelectric surfaces, therefore, eliminates the 
adhesion induced impulsive separation, resulting in symmetric positive and negative current half cycles. In other words, the non-contact mode operation of the TENG can potentially be used as a method of eliminating the asymmetry between positive and negative current half cycles, when subjected to a uniform and symmetric input motion. However, it should be noted that the output current is relatively lower in the non-contact mode due to reduced induction of electric fields.[27]

\subsection{Practical Implications of the Findings}

The findings of this study are directly applicable in enhancing the performance, reliability and sustainability of the TENGs in energy harvesting and self-powered sensing applications, as well as to develop standard methods for their characterisation and standardisation.

\subsubsection{TENGs for Sensing}

Considering self-powered sensing using TENGs, most of the applications presented in the literature for chemicals and radiation are composed of TENG structures with active materials sensitive to these stimuli.[16][21][17] At present, uniform motion inputs are used to operate these TENG devices, however, the current peaks corresponding to ascending and descending movements are somewhat variable (Fig. 1). Moreover, there are notable variations between the current peaks of the same polarity, especially for the peaks corresponding to the descending movement, that form the most significant portion of the outputs. Under such conditions, these devices are used to detect relatively significant variations of concentrations. Ideally, if a uniform current response can be obtained under a specified motion profile without undue variations between positive and negative peaks, or, within the peaks of a given polarity, this will ensure that the current output changes strictly corresponding to the change in the external stimuli, which in this case, the relevant chemical or radiation type. The author suggests that this sort of a scenario has the potential of extensively increasing the sensitivity and the reliability of the sensors, as even a small degree of variation in the current can be related to the change in the external stimuli. 
From the observations of this study, the criteria defined to minimize the variations of current peaks can be applied here; i.e. using the $I_{S C}$ in the non-contact mode of a TENG. Moreover, when this criterion is not feasible, utilising the $I_{S C}$ peaks corresponding to the ascending movement in the contact mode (of a uniform motion input) can be recommended. As indicated in Fig. 3b, the peaks corresponding to the ascending movement are consistent and repetitive, while providing relatively high peak values with respect to the non-contact mode. Moreover, these concepts are equally applicable to other sensing applications which depend on the current output of a TENG, such as motion sensing applications $[15,19,20]$, with the potential capability of enhancing their performance and reliability.

The significant differences in peak $I_{S C}$ during the ascending and descending TENG motion cycles could potentially be utilised for useful applications. For instance, this clear difference can be used as an indicator to evaluate the direction of the movement of a TENG, as the smaller $I_{S C}$ peaks tend to correspond to ascending movement, and the lager peaks to descending movement (under a uniform motion profile such as constant velocity or sinusoidal movements). This can further be extended to evaluate the polarity of the triboelectric charges on each TENG contact surface, providing useful information in addition to the polarity of the charge and current peaks.

\subsubsection{TENGs for Power Generation}

In terms of the power generation of the TENG, the asymmetric nature of current peaks could have a significant impact on the device performance. The uneven and sporadic nature of current outputs is consistently being highlighted as one of the key limitations of TENGs[33][34], as it causes significant variations in output power. These variations can disturb the operations of applications where TENGs are used as a direct power source, or, make it difficult to control output power via energy storage and other power management circuits. While these variations are mainly caused by the electrostatic induction of the TENG owing to the inherent distance-dependent electric field behaviour, the asymmetry between 
positive and negative current half cycles have a significant effect on intensifying the variations. For instance, the percentage variation of peak output power between positive and negative motion half cycles is depicted in Supplementary Note 4, indicating the variation in the contact mode would remain close to $80 \%$ in most of the load values. These variations could adversely affect the consistency of the TENG, resulting in increased crest factors.[33] In comparison, for the non-contact mode, this variation is around $10 \%$ for most of the load values. This indicates that the non-contact mode operation of the TENG reduces the overall variation between output power peaks, providing a relatively uniform peak power output (at the expense of a relatively lower peak power), when subjected to a uniform and symmetric input motion profile.

On the other hand, the asymmetry of current peaks due to the adhesion induced impulsive separation can potentially be used as an advantage. For instance, considering contact regime, for majority of the loads, the outputs corresponding to the descending movement are around $80 \%$ larger than the outputs related to ascending movement as described in the previous paragraph (Fig. S4). This, in other words, indicates an enhancement of TENG power output by $80 \%$ compared to ascending movement mainly due to the adhesion induced impulsive separation of TENG surfaces. Therefore, deriving methods to potentially enhance this effect would result in higher peak power outputs under a given motion profile, which would be specifically useful when enhanced instantaneous power outputs are required.

\subsubsection{Device Characterisation and Standardisation}

The findings of this study lead towards a timely and essential discussion on how we interpret the results of TENGs, their characterisation methods and the standards, as discussed in this section.

For instance, considering the characterising of $I_{S C}$, the normal practise is to obtain the peak values of the current, or, provide an average of peak currents corresponding to a specified motion profile (ex: peak current under $1 \mathrm{~mm}$ amplitude, $1 \mathrm{~Hz}$ frequency sinusoidal motion, 10 
$\mathrm{N}$ contact force). However, the mismatch of magnitude of the current peaks caused by the adhesion induced impulsive separation brings up the issue of which category of current peaks (positive, negative or highest) should be considered for the current characterisation. For instance, it was clear from section 2 and section 3 that the ascending movement of the TENG layers is relatively smooth and closely follows the specified reference motion profile.

Therefore, the resulting current peaks correspond to the ascending motion profile, while also being uniform and repetitive. On the other hand, the descending movement is altered by the adhesion induced impulsive separation, and, as proven in section 2, the resulting actual separation velocities far exceed the specified motion profile. Furthermore, as indicated by the experimental results in section 2 and section 3, these outputs are likely to be sporadic, impulsive and less repeatable. Hence, the observed outputs related to descending motion do not realistically correspond to the specified motion profile, and, their nature is irregular and difficult to reproduce with high consistency. As per this argument, it might be fair to assume that the characterisation should be done using the current peaks related to the ascending movement.

However, the counter argument in this scenario would be that the impulsive separation of the TENG layers is an integral part of the TENG materials and design architecture, therefore, the current pulses corresponding to the descending movement can be used as the preferred outputs without neglecting these high magnitude current pulses. In this case, it may be advisable to mention the variation between the current peaks, and, the actual velocity profile during descending movement, in comparison to the ascending movement. The other option is to obtain the output profile of the TENG using the non-contact mode operation, which again, can be challenging in experimental conditions due to relatively lower outputs, and, the difficulty in measuring the actual separation in comparison to the ideal contact position of TENG surfaces.[23] A similar set of concepts can be applied to characterise the power generation of the TENG, which also depends on the rate of movement of TENG surfaces. 
These measures are particularly critical in comparing novel triboelectric materials, device structures, and, when developing standards for characterising TENGs.

\subsection{Other Factors Affecting the Asymmetry}

Within the scope of this work, the inherent and potentially unavoidable material characteristic of the TENG layers (internal factor) causing the asymmetry of TENG current, in the form of adhesion induced compulsive separation, was discussed in detail. There are many external factors which could potentially cause the asymmetric nature of current outputs, that can be related to the structure of the TENG and the motion profile.

In our previous work, we demonstrated the effect of the rate of movement of TENG layers on the output behaviour of a TENG. [1][27] Higher rate of the movement of TENG layers cause rapid changes in the potential difference between electrodes, resulting in elevated current outputs, hence, higher peak currents. Therefore, if there is an asymmetry of the motion inputs between the contact and the separation movements of a TENG created by the device structure (intermediate factors) or the motion profile itself (external factors), or a combination of these factors, it would result in an asymmetric current output during positive and negative current half-cycles. A number of TENG structures have been presented in the past with spring assisted separation mechanisms [13][22] which can be suggested as examples for the asymmetry created by the device structure (intermediate factors). These devices would produce significantly higher current outputs during the separation phase of TENG surfaces, due to the releasing action of the spring, which creates higher velocities.[22] In addition, many of the unregulated natural movements in the environment such as the wind, wave energy etc. can act as irregular motion inputs for the TENG (external factors), which therefore would result in asymmetric TENG current output peaks.

Apart from the variations in motion profiles, in a different approach, Wang et al.[35] recently developed an equivalent circuit model that could produce similar asymmetry in voltages of a VCSTENG device. This model attributed the asymmetric output generation to the 
difference between voltages during contact phase and separation phase of TENG layers. In their work, the output voltage of a TENG was defined to contain two voltage elements, among which one depends on the separation of triboelectric contact surfaces whereas the other depends on the thickness of the dielectric TENG layer. A switch on-off function was used to derive the differences in the two voltage components during contact phase and separation phase, resulting in a high output voltage during separation phase compared to contact phase, which eventually causes the asymmetry between current peaks. Therefore, the circuit model presented by Wang et al.[35] could potentially be used as an alternative source to simulate the asymmetric voltage of a TENG. However, the way this model relates to the physical TENG parameters, and, if this is applicable to a wide range of TENG working conditions (e.g. for experimental $\mathrm{V}_{\mathrm{OC}}$, ISC) are yet to be demonstrated.

\section{Conclusions and Perspectives}

A comprehensive analysis on the asymmetry and the irregular nature of the current peaks of a TENG is presented in this study. Within the scope of this work, the current generation is studied using a regular sinusoidal input motion profile via the DDEF model simulations and corresponding experimental results, in order to analyse the internal factors affecting the asymmetry between current peaks. The cause of this asymmetry is attributed to the adhesion induced impulsive separation, which affects the TENG current output when operating in the contact regime, and, increases the magnitude of the current peak corresponding the descending movement of TENG contact surfaces.

These output trends of the current peaks can be utilised as a guidance to optimise the energy harvesting and sensing applications for TENGs. Several criteria have been suggested depending on the experimental and theoretical results of this study, to minimize the variation of the current and power outputs, and, to increase the output generation depending on the sensing or energy harvesting applications. These criteria depend on the contact or non-contact mode operation of the TENG, as well as the load conditions. 
The focus of this study is to provide a fundamental analysis on the internal factors affecting the asymmetric nature of current outputs, and, the nature of their impact on the current generation. This study initiates a series of interesting future perspectives into investigating the exact underlying reasons which control the adhesion between the triboelectric surfaces. Such findings, and, the potential methods of controlling these phenomena would enable improved control of the current outputs[36], allowing the asymmetry to be reduced for less output variations, or, to be increased targeting higher instantaneous outputs. Some of these prospects would include the study of the effect of different material systems, physical and chemical surface modifications, chemical and physical bond types etc. on the current behaviour.

Developments of this nature will enable the design and construction of more reliable, consistent and high performing TENG devices for sustainable energy harvesting and sensing applications.

\section{Supporting Information}

Supporting information is included as Supplementary Note 1 to 4.

\section{Acknowledgements}

The author acknowledges the support of the Loughborough University Doctoral Prize Fellowship for funding this project. This work was also supported by the UK EPSRC Joint University Industry Consortium for Energy (Materials) and Devices Hub (JUICED Hub) (EP/R023662/1). Author is grateful for Professor Upul Wijayantha (Loughborough University principle investigator of the JUICED Hub) for providing materials and space for some of this work and fruitful discussions. Furthermore, the author likes to thank Prof. Jeremy Coupland for useful discussions and comments on the manuscript, and, Prof. Stephanos Theodossiades and Jake Sandall for their support in preparing the experimental setup.

\section{Competing Interests}

The author declares no conflicts of interest.

\section{References}


[1] R.D.I.G. Dharmasena, S.R.P. Silva, Towards optimized triboelectric nanogenerators, Nano Energy. 62 (2019) 530-549. https://doi.org/10.1016/J.NANOEN.2019.05.057.

[2] R.D.I.G. Dharmasena, K.D.G.I. Jayawardena, Z. Saadi, X. Yao, R.M.I. Bandara, Y. Zhao, S.R.P. Silva, Energy Scavenging and Powering E-Skin Functional Devices, Proc. IEEE. (2019) 1-19. https://doi.org/10.1109/JPROC.2019.2929286.

[3] C. Wu, A.C. Wang, W. Ding, H. Guo, Z.L. Wang, Triboelectric Nanogenerator: A Foundation of the Energy for the New Era, Adv. Energy Mater. 9 (2019) 1802906. https://doi.org/10.1002/aenm.201802906.

[4] Z.L. Wang, Triboelectric nanogenerators as new energy technology and self-powered sensors - Principles, problems and perspectives, Faraday Discuss. 176 (2015) 447-458. https://doi.org/10.1039/c4fd00159a.

[5] S. Xu, W. Ding, H. Guo, X. Wang, Z.L. Wang, Boost the Performance of Triboelectric Nanogenerators through Circuit Oscillation, Adv. Energy Mater. 9 (2019) 1900772. https://doi.org/10.1002/aenm.201900772.

[6] Z.L. Wang, Triboelectric Nanogenerator (TENG)—Sparking an Energy and Sensor Revolution, Adv. Energy Mater. 10 (2020) 2000137.

[7] J. Chen, Z.L. Wang, Reviving Vibration Energy Harvesting and Self-Powered Sensing by a Triboelectric Nanogenerator, Joule. 1 (2017) 480-521. https://doi.org/10.1016/j.joule.2017.09.004.

[8] H. Zhang, Y. Yang, Y. Su, J. Chen, C. Hu, Z. Wu, Y. Liu, C. Ping Wong, Y. Bando, Z.L. Wang, Triboelectric nanogenerator as self-powered active sensors for detecting liquid/gaseous water/ethanol, Nano Energy. 2 (2013) 693-701. https://doi.org/10.1016/J.NANOEN.2013.08.004.

[9] W. Ding, J. Zhou, J. Cheng, Z. Wang, H. Guo, C. Wu, S. Xu, Z. Wu, X. Xie, Z.L. Wang, TriboPump: A Low-Cost, Hand-Powered Water Disinfection System, Adv. Energy Mater. 9 (2019) 1901320. 
[10] B. Dudem, R.D.I.G. Dharmasena, S.A. Graham, J.W. Leem, H. Patnam, A.R. Mule, S.R.P. Silva, J.S. Yu, Exploring theoretical and experimental optimization towards high-performance triboelectric nanogenerators using microarchitecture silk cocoon films, Nano Energy. (2020) 104882.

[11] R.D.I.G. Dharmasena, Triboelectric self-powered energy systems. [Doctoral Dissertation], University of Surrey, 2019.

[12] S. Wang, Y. Xie, S. Niu, L. Lin, C. Liu, Y.S. Zhou, Z.L. Wang, Maximum Surface Charge Density for Triboelectric Nanogenerators Achieved by Ionized-Air Injection: Methodology and Theoretical Understanding, Adv. Mater. 26 (2014) 6720-6728. https://doi.org/10.1002/adma.201402491.

[13] G. Zhu, Z.-H. Lin, Q. Jing, P. Bai, C. Pan, Y. Yang, Y. Zhou, Z.L. Wang, Toward Large-Scale Energy Harvesting by a Nanoparticle-Enhanced Triboelectric Nanogenerator, Nano Lett. 13 (2013) 847-853. https://doi.org/10.1021/n14001053.

[14] G. Zhu, C. Pan, W. Guo, C.-Y. Chen, Y. Zhou, R. Yu, Z.L. Wang, TriboelectricGenerator-Driven Pulse Electrodeposition for Micropatterning, Nano Lett. 12 (2012) 4960-4965. https://doi.org/10.1021/nl302560k.

[15] C. Bao Han, C. Zhang, X.H. Li, L. Zhang, T. Zhou, W. Hu, Z. Lin Wang, Self-powered velocity and trajectory tracking sensor array made of planar triboelectric nanogenerator pixels, Nano Energy. 9 (2014) 325-333. https://doi.org/10.1016/j.nanoen.2014.07.025.

[16] Z.-H. Lin, G. Zhu, Y.S. Zhou, Y. Yang, P. Bai, J. Chen, Z.L. Wang, A Self-Powered Triboelectric Nanosensor for Mercury Ion Detection, Angew. Chemie Int. Ed. 52 (2013) 5065-5069. https://doi.org/10.1002/anie.201300437.

[17] Z.-H. Lin, G. Cheng, Y. Yang, Y.S. Zhou, S. Lee, Z.L. Wang, Triboelectric Nanogenerator as an Active UV Photodetector, Adv. Funct. Mater. 24 (2014) 28102816. https://doi.org/10.1002/adfm.201302838.

[18] S.A. Nahian, R.K. Cheedarala, K.K. Ahn, A study of sustainable green current 
generated by the fluid-based triboelectric nanogenerator (FluTENG) with a comparison of contact and sliding mode, Nano Energy. 38 (2017) 447-456. https://doi.org/10.1016/j.nanoen.2017.06.012.

[19] H. Zhang, Y. Yang, Y. Su, J. Chen, K. Adams, S. Lee, C. Hu, Z.L. Wang, Triboelectric Nanogenerator for Harvesting Vibration Energy in Full Space and as Self-Powered Acceleration Sensor, Adv. Funct. Mater. 24 (2014) 1401-1407. https://doi.org/10.1002/adfm.201302453.

[20] F. Yi, L. Lin, S. Niu, J. Yang, W. Wu, S. Wang, Q. Liao, Y. Zhang, Z.L. Wang, SelfPowered Trajectory, Velocity, and Acceleration Tracking of a Moving Object/Body using a Triboelectric Sensor, Adv. Funct. Mater. 24 (2014) 7488-7494. https://doi.org/10.1002/adfm.201402703.

[21] Z.-H. Lin, Y. Xie, Y. Yang, S. Wang, G. Zhu, Z.L. Wang, Enhanced Triboelectric Nanogenerators and Triboelectric Nanosensor Using Chemically Modified $\mathrm{TiO}_{2}$ Nanomaterials, ACS Nano. 7 (2013) 4554-4560. https://doi.org/10.1021/nn401256w.

[22] J. Chen, G. Zhu, W. Yang, Q. Jing, P. Bai, Y. Yang, T.-C. Hou, Z.L. Wang, HarmonicResonator-Based Triboelectric Nanogenerator as a Sustainable Power Source and a Self-Powered Active Vibration Sensor, Adv. Mater. 25 (2013) 6094-6099. https://doi.org/10.1002/adma.201302397.

[23] R.D.I.G. Dharmasena, K.D.G.I. Jayawardena, C.A. Mills, J.H.B. Deane, J.V. Anguita, R.A. Dorey, S.R.P. Silva, Triboelectric nanogenerators: providing a fundamental framework, Energy Environ. Sci. 10 (2017) 1801-1811. https://doi.org/10.1039/C7EE01139C.

[24] S. Niu, S. Wang, L. Lin, Y. Liu, Y.S. Zhou, Y. Hu, Z.L. Wang, Theoretical study of contact-mode triboelectric nanogenerators as an effective power source, Energy Environ. Sci. 6 (2013) 3576-3583. https://doi.org/10.1039/c3ee42571a.

[25] S. Niu, Z.L. Wang, Theoretical systems of triboelectric nanogenerators, Nano Energy. 
14 (2015) 161-192. https://doi.org/10.1016/j.nanoen.2014.11.034.

[26] R.D.I.G. Dharmasena, K.D.G.I. Jayawardena, C.A. Mills, R.A. Dorey, S.R.P. Silva, A unified theoretical model for Triboelectric Nanogenerators, Nano Energy. 48 (2018) 391-400. https://doi.org/10.1016/j.nanoen.2018.03.073.

[27] R.D.I.G. Dharmasena, J.H.B. Deane, S.R.P. Silva, Nature of Power Generation and Output Optimization Criteria for Triboelectric Nanogenerators, Adv. Energy Mater. (2018) 1802190. https://doi.org/10.1002/aenm.201802190.

[28] J.H.B. Deane, R.D.I.G. Dharmasena, G. Gentile, Power computation for the triboelectric nanogenerator, Nano Energy. 54 (2018) 39-49. https://www.sciencedirect.com/science/article/pii/S2211285518307080 (accessed October 18, 2018).

[29] Z.L. Wang, On Maxwell's displacement current for energy and sensors: the origin of nanogenerators, Mater. Today. 20 (2017) 74-82. https://doi.org/10.1016/j.mattod.2016.12.001.

[30] M.K. Chaudhury, G.M. Whitesides, Direct Measurement of Interfacial Interactions between Semispherical Lenses and Flat Sheets of Poly(dimethylsiloxane) and Their Chemical Derivatives, Langmuir. 7 (1991) 1013-1025. https://doi.org/10.1021/la00053a033.

[31] B. Bhushan, Adhesion and stiction: Mechanisms, measurement techniques, and methods for reduction, J. Vac. Sci. Technol. B Microelectron. Nanom. Struct. 21 (2003) 2262-2296. https://doi.org/10.1116/1.1627336.

[32] R.R. Netz, D. Andelman, Neutral and charged polymers at interfaces, Phys. Rep. 380 (2003) 1-95. https://doi.org/10.1016/S0370-1573(03)00118-2.

[33] H. Ryu, J.H. Lee, U. Khan, S.S. Kwak, R. Hinchet, S.-W. Kim, Sustainable direct current powering a triboelectric nanogenerator via a novel asymmetrical design, Energy Environ. Sci. (2018). https://doi.org/10.1039/C8EE00188J. 


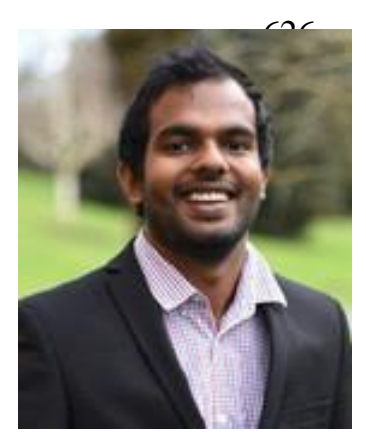

Dr Ishara Dharmasena is a Doctoral Prize Fellow at Wolfson School of Mechanical, Electrical and Manufacturing Engineering, Loughborough University. He received his B.Sc. (Engineering) degree from University of Moratuwa (2014), and, PhD degree from the Advanced Technology Institute (ATI), University of Surrey, specializing in energy harvesting

[34] X. Li, X. Yin, Z. Zhao, L. Zhou, D. Liu, C. Zhang, C. Zhang, W. Zhang, S. Li, J. Wang, Z.L. Wang, Long-Lifetime Triboelectric Nanogenerator Operated in Conjunction Modes and Low Crest Factor, Adv. Energy Mater. 10 (2020) 1903024. https://doi.org/10.1002/aenm.201903024.

[35] Z. Wang, W. Liu, J. Hu, W. He, H. Yang, C. Ling, Y. Xi, X. Wang, A. Liu, C. Hu, Two voltages in contact-separation triboelectric nanogenerator: from asymmetry to symmetry for maximum output, Nano Energy. (2020) 104452.

[36] R.D.I.G. Dharmasena, H.M. Cronin, R.A. Dorey, S.R.P. Silva, Direct current contactmode triboelectric nanogenerators via systematic phase shifting, Nano Energy. (2020) 104887. https://doi.org/10.1016/j.nanoen.2020.104887.
631

632

633

and wearable electronics (2019). He has previously worked in the ATI as a research fellow, and, at Sri Lanka Institute of Nanotechnology (SLINTEC) as a research scientist. His research interests include mechanical energy harvesting, triboelectric nanogenerators, wearable electronics and flexible electronics. 